\title{
GROWTH AND YIELD PARAMETERS OF SORGHUM GENOTYPES AS AFFECTED BY ARTIFICIAL INOCULATION TECHNIQUES FOR SCREENING AGAINST HEAD SMUT IN NIGERIA
}

\author{
Fagwalawa ${ }^{1}$, L.D., M.T. ${ }^{1}$ Yakasai and ${ }^{*}$ Kutama $^{2}$, A.S. \\ ${ }^{1}$ Department of Biology, Kano University of Science and Technology, Wudil \\ ${ }^{2}$ Department of Biological Science, Faculty of Sciences, Federal University, Dutse, Jigawa State \\ *Correspondence author: kutamasak@yahoo.com
}

\begin{abstract}
Field trial was conducted at Bayero University, Kano research farm with the aim of determining the effect of stem injection artificial inoculation technique on the growth and yield parameters of one hundred and four sorghum genotypes against head smut. The trial was laid on a randomized complete block design with two replications. Results obtained on the growth parameters which includes plant height, number of leaves and number of tillers per plant have shown that, of the 104 sorghum genotypes screened for head smuts, there was marked reduction in growth parameters in 27 (diseased) plants compared to non- infected (77) plants. Similarly, number of days to $50 \%$ heading as well as grain yield/hectare were significantly $(P<0.05)$ affected in head smut infected plants than in healthy plants, with the former usually having less number of days to heading, as well as less yield than the latter. However, days to $50 \%$ flowering was not significantly $(P>0.05)$ affected even among the diseased plants. The present study have shown that in head smut, plants showing symptoms usually matured earlier and produced less grain yield.

Keywords; sorghum genotypes, head smut, growth and yield parameters, Sudan savanna
\end{abstract}

\section{INTRODUCTION}

Dependence upon plants by humans for their very existence has been traced as far back as more than 13,000 years ago (Singh, 2005) and most of the human food supply worldwide is derived from not more than 20 crops namely: banana, plantain, barley, cassava, citrus, coconut, corn (maize), oats, peanut, pineapple, potato, pulses (beans, peas), rice, rye, sorghum, soybean, sugar beet, sugarcane, sweet potato, wheat and yam (Ahliawate, 2007). In the semi-arid Africa, sorghum is an important food crop for more than 150 million persons, and is usually grown in even nutrient depleted soil and harsh climatic conditions where other crops like maize and rice cannot thrive (Kutama, 2012).

Sorghum (Sorghum bicolor (L) Moench) is one of the world's major food crops, particularly in areas of high temperature and low rainfall. Global production is estimated to exceed 40 million hectares, ranking it fifth in importance among cereals (FAO, 2010). It is relatively drought tolerant and can therefore be grown in marginal, semi-arid areas where rainfall is unreliable and the cultivation of food crops such as maize is not feasible. Sorghum is very versatile as a food and feed grain and exhibits wide adaptation and yield stability in marginal areas where it is used as a subsistence staple food. As a consequence, sorghum is important in traditional, low input, cereal based, semi-arid production farming systems in Africa (Norman, et al., 1984).

Many techniques of artificial inoculation in both loose and head smuts have been suggested by many workers. It is however apparent that the appropriate inoculation procedure depends on the route of infection of a particular pathogen and the developmental stage of the host plant at which it is most susceptible. These, plus the question of whether small or large number of sorghum lines is used for the inoculation, partly decide which method mostly fits. One of the conventional as well as accepted of these artificial inoculation techniques is the stem injection (Kutamaet al., 2011a, b, c) artificial inoculation used in head smuts screening.

This method has been found suitable for both loose and head smuts. Keayet al. (1969) showed that $64 \%$ of 3 - 4 weeks old sorghum plants became infected with loose smut when plants were inoculated with aid of a hypodermic syringe. It is noted that King (1969) employed this technique for screening sorghum collections in Nigeria. However, it is noted that the different inoculation techniques possess different effect on the resultant growth parameters of various sorghum genotypes. This paper report the effect of stem injection artificial inoculation used for screening sorghum genotype against head smut, on the growth and yield of sorghum genotypes in Nigeria.

\section{MATERIALS AND METHODS}

Field screening was conducted on research farm of the Faculty of Agriculture, Bayero University, Kano located on $11^{\circ} 58.981 \mathrm{~N}, 008^{\circ} 25.298 \mathrm{E}$ and on $454 \mathrm{~m}$ elevation during the 2010 growing season.

Randomized complete block design (RCBD) field experiment was conducted. The layout involved two blocks each.

The plot size was $1.5 \mathrm{~m}$ long by three ridges each of $75 \mathrm{~cm}$ apart and one sorghum line/accession was sown per plot at the rate of $3 \mathrm{~kg} / \mathrm{ha}$, three seeds per hole and $0.4 \mathrm{~m}$ plant spacing (Komolafeet al., 1985). 
Each block therefore was comprising of 104 plots and therefore containing the 104 sorghum lines. This was randomized in the second block for each of the two diseases. Sowing was done on 8th to 11th July, 2010.

\section{Agronomic practices}

One week after germination, seedlings were thinned to one plant per stand. This resulted in about 15 to 18 plants per plot. First weeding was done manually at 14 days after germination while the second weeding was done four weeks after germination because there was rapid regeneration of weeds two weeks after the first weeding.

Compound fertilizer (NPK, 20:10:10) was applied at the rate of $50 \mathrm{~kg} / \mathrm{ha}$ (Louis et al., 2007) in two equal split doses, one after the first weeding and the next at booting stage.

\section{Artificial Inoculation \\ Stem Injection Technique}

Three weeks after sowing (3WAS), $0.5 \mathrm{~g}$ of previously collected, dried and stored teliospores of $S$. reilianum(head smut) were germinated separately in 1 liter of distilled water each for 28 hours and blended for 30 seconds at the speed of 500r/min, using an electric blender in order to obtain a homogenous teliospore suspension. One milliliter of the suspension was introduced into the main stem of the plant with a pediatric syringe by inserting the needle gently into the stem or growing point while carefully holding and supporting the whole plant with a hand to prevent damage. The same procedure was repeated 40 days after sowing (40DAS) where injection of spore suspension was also done at stem or growing point. The plants in both cases were allowed to grow normally up to physiological maturity.

Plant height $(\mathrm{cm})$ was measured at 2 weeks interval after inoculation (WAI) up to 10 weeks after inoculation (WAI). The height was determined by carefully measuring from the base/ground level to the apex or the growing point using a measuring tape. At least two plants were measured from the middle row and the average was taken per plot. The number of leaves per plant was counted from two weeks after inoculation up to $8 \mathrm{WAI}$ at 2-week intervals. The number of tillers per plant was counted fortnightly two weeks after inoculation up to 8 WAI.

The number of days to $50 \%$ flowering in a plot was determined by monitoring the number of days when $50 \%$ of the plants in each plot came into booting stage. This was calculated from the days after sowing. Number of days to $50 \%$ heading was determined by monitoring the number of days when $50 \%$ of the sorghum heads in each plot had produced grains. This was calculated from the days after sowing. When the various cultivars had attained physiological maturity, the sorghum heads from the inner row in each plot were cut, sun dried, threshed and winnowed manually. The grains were weighed. The figures were later converted to kilograms per hectare.

\section{RESULTS AND DISCUSSION}

Table 1 shows the growth parameters which include plant height, number of leaves, number of tillers per plant from 2 weeks after inoculation (2WAI) to 8WAI.
Plant height significantly varied $(P<0.05)$ among sorghum genotypes progressively from 2 WAI to 4WAI but the differences were not significant from 6WAI to 8 WAI. By the end of 8WAI, sorghum genotype SSV2008013 was the shortest $(102.0 \mathrm{~cm})$ while SSV2006045 was the tallest $(167.2 \mathrm{~cm})$. However, there was progressive increase in plant height across the growing period.Similarly, the mean number of tillers per plant significantly varied from 2WAI to 8WAI i. e. throughout the period when data was been taken. Significant difference at $5 \%$ probability level was also observed on the mean number of leaves per plant from 2 WAI to 4 WAI, after which the differences were no longer significant.

The variations in the growth and yield parameters of the diseased plants could be due to differences in the response of the different genotypes to head smut pathogen. Significant reduction in plant height and increased tilleringwas only and earlier on demonstrated by Tarr (1962) in head smutted plants with variations among cultivars but not in head smutted plants. Many authors did not report any shoot stunting in head smutted plants (Kutamaet al., 2011a). Matheussenet al. (1991) noted that some aspects of head smut in sorghum suggest that plant hormones are involved in the disease. Frederiksen (1977) had noted that head smut causes a substantial reduction in plant height and this effect was documented later by Naidooet al. (1991). The occurrence of reduction in height and other growth and yield parameters in most diseased sorghum genotypes in both head smut infected plants observed in present study shows that plant height and other parameters such as tiller number, days to flowering and heading as well as grain yield are important components of the smut diseases. Matheussenet al. (1991) attributed these reductions in growth and yield due to $\mathrm{GA}_{3}$ production in the diseased plant which was earlier noted by Phinney and Spray (1982) who showed that both plant height and sexual development are altered in head smut-infected maize mutants with blocks in their $\mathrm{GA}_{3}$ biosynthetic pathway. However, the mechanism of GAs in growth reduction is not very clear. According to these authors, when symptoms are less severe (partial infection), the production of $\mathrm{GA}_{3}$ by the panicle is less affected and dwarfing is not severe. This hypothesis does not exclude the possibility that GAs are also made in the nodes and internodes and thereby influence elongation. $\mathrm{GA}_{3}$ application to sorghum inhibits tiller production (Morgan et al., 1977). Similarly, the great variations in growth parameters of the different varieties might be due to their origin and adaptation. While most genotypes grown in the Sahel and Sudan savanna AEZs tend to mature within 3-5 months, cultivars grown in the Guinea savanna AEZs mature in 4 -7 months period. Another reason for the difference in growth and yield parameters was probably due to late sowing. Early planting has been shown to increase both growth and yield parameters in all crops (David and Adam, 1998) as such late planting may results into reduced growth and yield characters as obtained in this study (Kutama et al.,2011b). 
Table 1. Mean plant height (cm), number of tillers and number of leaves/ plant of sorghum genotypes screened for resistance to head smut disease

\begin{tabular}{|c|c|c|c|c|c|c|c|c|c|c|c|c|c|}
\hline \multirow[t]{3}{*}{$\mathbf{S / N}$} & \multirow{3}{*}{$\begin{array}{l}\text { Sorghum } \\
\text { Genotype }\end{array}$} & \multicolumn{11}{|c|}{ Growth parameters at: } & \multirow[b]{3}{*}{ Lfno. } \\
\hline & & \multicolumn{3}{|l|}{ 2WAI } & \multicolumn{3}{|l|}{ 4WAI } & \multicolumn{3}{|l|}{ 6WAI } & \multicolumn{2}{|l|}{ 8WAI } & \\
\hline & & Plht & TIno & Lfno. & Plht & TIno & Lfno. & Plht & TIno & Lfno. & Plht & TIno & \\
\hline 1 & SSV2006002 & 89.45 & 0.0 & 8.0 & 92.65 & 0.0 & 9.0 & 121.1 & 0 & 10 & 130.2 & 0.5 & 12.0 \\
\hline 2 & SSV2006006 & 73.9 & 0.0 & 7.0 & 90.45 & 0.0 & 9.0 & 90.1 & 0 & 8 & 120.2 & 1.0 & 12.0 \\
\hline 3 & SSV2006007 & 88.3 & 0.0 & 8.0 & 93.3 & 0.0 & 9.0 & 109.2 & 0 & 9 & 123.4 & 0.5 & 12.0 \\
\hline 4 & SSV2006011 & 87.2 & 0.0 & 8.0 & 97.3 & 0.0 & 11.0 & 121.1 & 0 & 11 & 132.0 & 1.0 & 11.0 \\
\hline 5 & SSV2006013 & 78.85 & 0.0 & 8.0 & 89.3 & 0.0 & 11.0 & 111.3 & 0 & 10 & 123.4 & 0.5 & 11.5 \\
\hline 6 & SSV2006014 & 67.95 & 0.0 & 8.0 & 80.2 & 0.0 & 9.0 & 113.3 & 0 & 11 & 124.5 & 1.0 & 12.0 \\
\hline 7 & SSV2006015 & 107.35 & 0.0 & 9.0 & 120.1 & 0.0 & 9.0 & 125.3 & 0 & 10 & 140.2 & 0.5 & 12.0 \\
\hline 8 & SSV2006016 & 84.55 & 0.0 & 7.5 & 90.2 & 0.0 & 9.0 & 102.1 & 0 & 9.5 & 145.0 & 1.0 & 12.0 \\
\hline 9 & SSV2006017 & 98.3 & 0.0 & 8.0 & 105.3 & 0.0 & 8.5 & 140.3 & 0 & 8 & 167.3 & 0.5 & 12.0 \\
\hline 10 & SSV2006018 & 105.15 & 2.0 & 7.0 & 113.3 & 2.0 & 9.0 & 121.1 & 2 & 8 & 134.2 & 2.0 & 11.5 \\
\hline 11 & SSV2006021 & 65.3 & 1.5 & 7.0 & 80.7 & 1.5 & 9.0 & 99.3 & 1 & 8 & 132.0 & 1.0 & 11.0 \\
\hline 12 & SSV2006024 & 69.05 & 0.0 & 7.0 & 80.4 & 0.0 & 9.5 & 97.5 & 0 & 9 & 121.0 & 1.0 & 11.5 \\
\hline 13 & SSV2006026 & 63.5 & 0.0 & 7.0 & 78.6 & 0.0 & 10.0 & 98.8 & 0 & 9 & 112.1 & 0.5 & 11.5 \\
\hline 14 & SSV2006027 & 62.5 & 1.0 & 8.0 & 80.1 & 1.0 & 11.5 & 98.3 & 0 & 9 & 102.1 & 0.5 & 11.5 \\
\hline 15 & SSV2006029 & 74.3 & 0.0 & 8.0 & 81.3 & 0.0 & 10.5 & 99.3 & 0 & 8 & 102.4 & 0.0 & 11.5 \\
\hline 16 & SSV2006030 & 73.5 & 0.0 & 7.0 & 83.1 & 0.0 & 10.5 & 93.2 & 0 & 8 & 121.0 & 0.0 & 11.5 \\
\hline 17 & SSV2006031 & 78.8 & 0.0 & 7.0 & 87.2 & 0.0 & 10.5 & 98.0 & 0 & 8 & 100.9 & 0.0 & 11.5 \\
\hline 18 & SSV2006033 & 45.9 & 1.0 & 7.0 & 65.3 & 1.0 & 11.0 & 99.1 & 2 & 8 & 114.1 & 1.0 & 11.5 \\
\hline 19 & SSV2006035 & 86.5 & 0.5 & 7.0 & 95.3 & 0.5 & 10.5 & 102.9 & 1 & 8 & 132.1 & 0.5 & 12.0 \\
\hline 20 & SSV2006036 & 63.75 & 0.5 & 7.0 & 80.0 & 0.5 & 9.0 & 103.3 & 1 & 7 & 123.1 & 1.0 & 12.0 \\
\hline 21 & SSV2006039 & 62.95 & 2.0 & 8.0 & 78.1 & 2.0 & 8.5 & 111.1 & 2 & 7.5 & 123.1 & 2.0 & 12.0 \\
\hline 22 & SSV2006041 & 62.6 & 0.0 & 7.5 & 71.1 & 0.0 & 9.0 & 98.3 & 0 & 8 & 104.3 & 0.5 & 12.0 \\
\hline 23 & SSV2006045 & 98.68 & 0.0 & 9.0 & 102.0 & 0.0 & 10.0 & 132.1 & 0 & 8.5 & 167.2 & 1.0 & 12.0 \\
\hline 24 & SSV2006047 & 71.15 & 1.0 & 8.0 & 79.9 & 1.0 & 10.5 & 121.0 & 1 & 8 & 135.4 & 1.0 & 12.0 \\
\hline 25 & SSV2008001 & 65.9 & 0.0 & 8.0 & 76.2 & 0.0 & 9.0 & 98.8 & 0 & 9 & 123.2 & 0.0 & 12.0 \\
\hline 26 & SSV2008002 & 67.9 & 0.0 & 7.5 & 67.2 & 0.0 & 9.0 & 100.2 & 0 & 8 & 123.1 & 0.0 & 12.0 \\
\hline 27 & SSV2008004 & 68.3 & 0.0 & 7.5 & 67.2 & 0.0 & 9.0 & 105.2 & 0 & 8 & 115.5 & 0.0 & 12.0 \\
\hline 28 & SSV2008005 & 66.3 & 0.0 & 7.5 & 65.1 & 0.0 & 9.0 & 123.2 & 0 & 8 & 142.1 & 0.0 & 11.5 \\
\hline 29 & SSV2008006 & 63.3 & 2.5 & 7.5 & 69.1 & 2.5 & 9.0 & 123.0 & 2 & 9 & 134.2 & 1.0 & 11.0 \\
\hline 30 & SSV2008007 & 65.0 & 0.0 & 8.0 & 69.0 & 0.0 & 9.0 & 98.0 & 0 & 8 & 121.1 & 0.0 & 10.5 \\
\hline 31 & SSV2008008 & 68.2 & 0.0 & 8.0 & 78.1 & 0.0 & 9.0 & 97.3 & 0 & 8 & 124.9 & 0.5 & 11.5 \\
\hline 32 & SSV2008009 & 61.45 & 0.0 & 8.0 & 77.2 & 0.0 & 9.5 & 98.0 & 0 & 7 & 123.3 & 0.0 & 12.0 \\
\hline 33 & SSV2008010 & 54.85 & 2.0 & 8.0 & 79.1 & 2.0 & 10.0 & 102.1 & 2 & 8 & 120.9 & 1.5 & 12.0 \\
\hline 34 & SSV2008012 & 55.55 & 0.0 & 8.0 & 68.1 & 0.0 & 10.0 & 121.2 & 0 & 7 & 143.2 & 0.0 & 11.0 \\
\hline 35 & SSV2008013 & 64.7 & 0.0 & 8.0 & 65.2 & 0.0 & 10.0 & 112.1 & 0 & 8 & 123.1 & 0.0 & 11.0 \\
\hline 36 & SSV2008017 & 65.45 & 0.0 & 8.0 & 68.2 & 0.0 & 10.0 & 98.3 & 0 & 7 & 102.0 & 0.5 & 11.0 \\
\hline \multirow[t]{2}{*}{37} & SSV2008018 & 72.55 & 0.0 & 8.0 & 82.3 & 0.0 & 10.0 & 98.4 & 0 & 8 & 103.3 & 0.0 & 10.5 \\
\hline & \multicolumn{13}{|c|}{146} \\
\hline
\end{tabular}


Table 1 continue

\begin{tabular}{|c|c|c|c|c|c|c|c|c|c|c|c|c|c|}
\hline \multirow[t]{3}{*}{$\mathbf{S / N}$} & \multirow{3}{*}{$\begin{array}{l}\text { Sorghum } \\
\text { Genotype }\end{array}$} & \multicolumn{11}{|c|}{ Growth parameters at: } & \multirow[b]{3}{*}{ Lfno. } \\
\hline & & \multicolumn{3}{|l|}{ 2WAI } & \multicolumn{3}{|l|}{ 4WAI } & \multicolumn{3}{|l|}{ 6WAI } & \multicolumn{2}{|l|}{ 8WAI } & \\
\hline & & Plht & TIno & Lfno. & Plht & TIno & Lfno. & Plht & TIno & Lfno. & Plht & TIno & \\
\hline 38 & SSV2008019 & 67.55 & 0.0 & 8.0 & 68.2 & 0.0 & 10.0 & 98.9 & 0 & 7 & 114.4 & 0.0 & 11.0 \\
\hline 39 & SSV2008021 & 74.75 & 0.0 & 8.0 & 63.2 & 0.0 & 9.5 & 90.0 & 0 & 8 & 117.9 & 0.5 & 11.0 \\
\hline 40 & SSV2008022 & 62.25 & 0.0 & 8.0 & 65.2 & 0.0 & 9.0 & 87.3 & 0 & 9 & 119.0 & 0.5 & 11.0 \\
\hline 41 & SSV2008023 & 46.45 & 1.5 & 8.0 & 76.1 & 1.5 & 9.0 & 98.9 & 1 & 7 & 117.0 & 1.0 & 11.5 \\
\hline 42 & SSV2008025 & 62.5 & 0.0 & 8.0 & 58.1 & 0.0 & 9.0 & 99.8 & 0 & 7 & 113.0 & 0.5 & 12.0 \\
\hline 43 & SSV2008026 & 71.95 & 0.0 & 8.0 & 60.9 & 0.0 & 10.0 & 98.0 & 0 & 7 & 120.1 & 0.5 & 12.0 \\
\hline 44 & SSV2008028 & 61.45 & 0.0 & 8.0 & 65.1 & 0.0 & 10.0 & 123.3 & 0 & 7 & 143.2 & 1.0 & 12.0 \\
\hline 45 & SSV2008029 & 51.95 & 1.0 & 8.0 & 62.1 & 1.0 & 10.0 & 101.2 & 1 & 8 & 123.3 & 0.5 & 12.0 \\
\hline 46 & SSV2008030 & 57.40 & 0.0 & 8.0 & 69.2 & 0.0 & 10.0 & 98.3 & 0 & 8 & 104.2 & 1.0 & 12.0 \\
\hline 47 & SSV2008031 & 66.65 & 0.0 & 8.0 & 73.2 & 0.0 & 10.0 & 102.0 & 0 & 8 & 123.2 & 0.5 & 12.0 \\
\hline 48 & SSV2008032 & 47.1 & 0.0 & 8.0 & 99.0 & 0.0 & 11.0 & 103.9 & 0 & 10 & 132.2 & 0.5 & 12.0 \\
\hline 49 & SSV2008033 & 58.9 & 0.0 & 7.0 & 81.1 & 0.0 & 10.0 & 89.3 & 0 & 9 & 109.2 & 0.5 & 12.0 \\
\hline 50 & SSV2008034 & 49.45 & 0.5 & 7.5 & 62.1 & 0.5 & 9.0 & 111.1 & 0 & 9 & 124.4 & 0.5 & 12.0 \\
\hline 51 & SSV2008035 & 54.3 & 0.0 & 7.0 & 77.2 & 0.0 & 9.0 & 99.0 & 0 & 10 & 121.0 & 0.0 & 12.0 \\
\hline 52 & SSV2008036 & 65.6 & 0.0 & 7.0 & 74.1 & 0.0 & 9.0 & 123.3 & 0 & 10 & 140.3 & 0.5 & 11.0 \\
\hline 53 & SSV2008039 & 65.4 & 1.0 & 7.0 .0 & 67.1 & 1.0 & 9.0 & 112.2 & 0 & 10 & 132.8 & 0.0 & 11.0 \\
\hline 54 & SSV2008040 & 87.9 & 0.0 & 7.5 & 65.2 & 0.0 & 9.0 & 132.2 & 0 & 10 & 132.2 & 0.0 & 11.0 \\
\hline 55 & SSV2008041 & 65.9 & 0.0 & 7.0 & 77.1 & 0.0 & 9.0 & 100.0 & 0 & 10 & 123.3 & 0.0 & 10.0 \\
\hline 56 & SSV2008042 & 57.05 & 0.0 & 7.0 & 67.1 & 0.0 & 9.0 & 123.1 & 0 & 9.5 & 134.3 & 0.0 & 10.0 \\
\hline 57 & SSV2008044 & 68.05 & 0.0 & 7.0 & 64.2 & 0.0 & 9.0 & 121.0 & 0 & 9 & 143.2 & 0.0 & 11.0 \\
\hline 58 & SSV2008046 & 65.5 & 1.0 & 7.0 & 72.1 & 1.0 & 9.0 & 107.4 & 0 & 9 & 123.1 & 0.0 & 12.0 \\
\hline 59 & SSV2008047 & 58.15 & 0.0 & 7.0 & 74.2 & 0.0 & 9.0 & 123.9 & 0 & 9 & 143.3 & 0.5 & 11.5 \\
\hline 60 & SSV2008048 & 51.5 & 0.0 & 7.0 & 92.1 & 0.0 & 9.0 & 134.0 & 0 & 9 & 156.0 & 0.0 & 11.0 \\
\hline 61 & SSV2008049 & 70.75 & 0.0 & 7.0 & 77.1 & 0.0 & 9.0 & 111.1 & 0 & 9 & 113.7 & 0.5 & 11.5 \\
\hline 62 & SSV2008051 & 54.95 & 0.0 & 7.0 & 67.1 & 0.0 & 9.0 & 102.3 & 0 & 9 & 123.3 & 0.5 & 11.5 \\
\hline 63 & SSV2008052 & 54.151 & 0.0 & 7.0 & 64.2 & 0.0 & 9.0 & 105.9 & 0 & 9 & 111.2 & 0.5 & 11.0 \\
\hline 64 & SSV2008053 & 63.05 & 0.0 & 7.0 & 74.2 & 0.0 & 9.0 & 112.9 & 0 & 10 & 123.9 & 0.5 & 12.0 \\
\hline 65 & SSV2008054 & 66.55 & 0.0 & 7.0 & 85.2 & 0.0 & 9.0 & 110.1 & 0 & 10 & 123.2 & 0.0 & 12.0 \\
\hline 66 & SSV2008055 & 81.55 & 0.0 & 7.5 & 78.8 & 0.0 & 9.0 & 123.0 & 0 & 10 & 134.4 & 0.5 & 12.0 \\
\hline 67 & SSV2008056 & 64.55 & 0.0 & 7.0 & 68.9 & 0.0 & 10.5 & 123.9 & 0 & 11 & 143.2 & 0.5 & 12.0 \\
\hline 68 & SSV2008057 & 94.45 & 0.5 & 7.0 & 64.9 & 0.5 & 10.0 & 143.5 & 0 & 10 & 156.0 & 0.5 & 12.0 \\
\hline 69 & SSV2008058 & 51.3 & 0.0 & 7.0 & 76.2 & 0.0 & 9.5 & 115.3 & 0 & 10 & 134.2 & 0.0 & 10.0 \\
\hline 70 & SSV2008059 & 68.03 & 0.0 & 7.0 & 89.2 & 0.0 & 9.5 & 123.3 & 0 & 10 & 123.0 & 0.5 & 12.0 \\
\hline 71 & SSV2008061 & 72.35 & 0.5 & 7.5 & 87.1 & 0.5 & 10.5 & 121.2 & 0 & 9 & 145.9 & 0.5 & 12.0 \\
\hline 72 & SSV2008063 & 65.6 & 0.0 & 7.0 & 89.9 & 0.0 & 9.0 & 121.0 & 0 & 9 & 145.2 & 0.0 & 10.0 \\
\hline 73 & SSV2008064 & 60.15 & 0.0 & 7.0 & 65.2 & 0.0 & 9.0 & 98.2 & 0 & 9 & 121.0 & 0.0 & 11.0 \\
\hline 74 & SSV2008066 & 57.5 & 1.0 & 7.0 & 89.7 & 1.0 & 9.0 & 121.9 & 1 & 9 & 145.2 & 0.5 & 12.0 \\
\hline
\end{tabular}




\section{Table 1 continue}

\begin{tabular}{|c|c|c|c|c|c|c|c|c|c|c|c|c|c|}
\hline \multirow[t]{3}{*}{$\mathbf{S / N}$} & \multirow{3}{*}{$\begin{array}{l}\text { Sorghum } \\
\text { Genotype }\end{array}$} & \multicolumn{11}{|c|}{ Growth parameters at: } & \\
\hline & & \multicolumn{3}{|l|}{ 2WAI } & \multicolumn{3}{|l|}{ 4WAI } & \multicolumn{3}{|l|}{ 6WAI } & \multicolumn{3}{|l|}{ 8WAI } \\
\hline & & Plht & TIno & Lfno. & Plht & TIno & Lfno. & Plht & TIno & Lfno. & Plht & TIno & Lfno. \\
\hline 75 & SSV2008067 & 65.0 & 1.0 & 7.0 & 91.1 & 1.0 & 9.0 & 132.1 & 2 & 9 & 145.8 & 1.0 & 12.0 \\
\hline 76 & SSV2008070 & 81.85 & 0.0 & 8.0 & 66.6 & 0.0 & 9.0 & 111.1 & 0 & 10 & 143.2 & 0.0 & 11.5 \\
\hline 77 & SSV2008072 & 72.3 & 0.0 & 7.0 & 68.9 & 0.0 & 9.0 & 89.3 & 0 & 10 & 121.0 & 0.0 & 11.0 \\
\hline 78 & SSV2008074 & 79.8 & 0.0 & 7.5 & 78.9 & 0.0 & 9.0 & 123.1 & 0 & 10 & 145.9 & 0.0 & 11.0 \\
\hline 79 & SSV2008075 & 62.05 & 0.0 & 8.0 & 78.3 & 0.0 & 0.0 & 118.3 & 0 & 11 & 132.2 & 0.0 & 11.0 \\
\hline 80 & SSV2008076 & 77.5 & 0.0 & 8.0 & 78.2 & 0.0 & 9.5 & 123.0 & 0 & 10 & 154.8 & 0.0 & 11.0 \\
\hline 81 & SSV2008078 & 82.0 & 0.0 & 8.0 & 87.3 & 0.0 & 10.0 & 112.0 & 0 & 9 & 156.3 & 0.5 & 11.5 \\
\hline 82 & SSV2008079 & 57.05 & 0.5 & 8.0 & 67.4 & 0.0 & 10.0 & 110.0 & 0 & 9 & 148.4 & 0.5 & 12.0 \\
\hline 83 & SSV2008080 & 58.55 & 0.0 & 7.0 & 77.2 & 0.0 & 9.5 & 102.0 & 0 & 10 & 132.1 & 0.5 & 12.0 \\
\hline 84 & SSV2008082 & 66.6 & 0.0 & 8.0 & 78.4 & 0.5 & 9.0 & 103.3 & 0 & 9 & 127.3 & 0.5 & 10.0 \\
\hline 85 & SSV2008084 & 66.6 & 1.5 & 8.0 & 65.3 & 1.5 & 9.5 & 104.3 & 1 & 9 & 134.2 & 1.0 & 11.0 \\
\hline 86 & SSV2008085 & 65.25 & 2.0 & 8.0 & 57.4 & 2.0 & 10.0 & 109.2 & 2 & 9 & 121.0 & 2.0 & 11.0 \\
\hline 87 & SSV2008086 & 76.55 & 1.5 & 8.0 & 87.3 & 1.5 & 9.0 & 98.2 & 2 & 9 & 112.1 & 1.5 & 11.0 \\
\hline 88 & SSV2008087 & 65.5 & 1.0 & 8.0 & 76.2 & 1.0 & 10.0 & 98.0 & 2 & 9 & 134.2 & 2.0 & 11.0 \\
\hline 89 & SSV2008088 & 67.55 & 1.0 & 8.0 & 65.2 & 1.0 & 11.0 & 120.1 & 2 & 9 & 133.3 & 1.5 & 11.0 \\
\hline 90 & SSV2008089 & 66.35 & 0.0 & 8.0 & 78.2 & 0.0 & 9.5 & 98.3 & 0 & 9 & 132.1 & 1.0 & 10.5 \\
\hline 91 & SSV2008090 & 57.1 & 1.5 & 8.0 & 65.3 & 1.5 & 9.5 & 112.1 & 2 & 10 & 123.1 & 1.5 & 12.0 \\
\hline 92 & SSV2008094 & 50.0 & 0.0 & 8.0 & 57.4 & 0.0 & 10.5 & 99.0 & 0 & 10 & 112.0 & 1.0 & 12.0 \\
\hline 93 & SSV2008096 & 64.55 & 0.0 & 8.0 & 78.3 & 0.0 & 9.5 & 102.9 & 0 & 10 & 130.0 & 1.0 & 12.0 \\
\hline 94 & SSV2008100 & 65.2 & 0.0 & 8.0 & 76.2 & 0.0 & 10.0 & 121.0 & 0 & 9 & 148.0 & 0.5 & 12.0 \\
\hline 95 & SSV2008101 & 54.05 & 1.5 & 8.0 & 65.2 & 1.5 & 10.0 & 112.2 & 2 & 9 & 127.8 & 1.5 & 12.0 \\
\hline 96 & SSV2008107 & 48.65 & 0.0 & 8.0 & 78.2 & 0.0 & 9.5 & 98.9 & 0 & 9 & 132.2 & 1.0 & 12.0 \\
\hline 97 & SSV2008110 & 58.2 & 0.0 & 7.0 & 72.1 & 0.0 & 9.5 & 111.9 & 0 & 9 & 123.3 & 0.0 & 11.0 \\
\hline 98 & SSV2008111 & 45.55 & 0.0 & 8.0 & 67.2 & 0.0 & 9.0 & 124.3 & 0 & 9 & 156.3 & 1.0 & 11.5 \\
\hline 99 & SSV2008112 & 74.35 & 1.0 & 7.0 & 87.1 & 1.0 & 9.0 & 111.0 & 1 & 10 & 163.1 & 0.5 & 11.5 \\
\hline 100 & SSV2008113 & 57.0 & 0.5 & 8.0 & 68.3 & 0.0 & 9.0 & 134.5 & 0 & 10 & 168.3 & 1.0 & 11.0 \\
\hline 101 & SSV2008116 & 65.5 & 0.0 & 7.0 & 76.2 & 0.0 & 9.5 & 124.5 & 0 & 11 & 146.3 & 1.0 & 12.0 \\
\hline 102 & SSV2008117 & 67.6 & 0.0 & 8.0 & 77.2 & 0.0 & 10.0 & 124.4 & 0 & 10 & 134.3 & 0.5 & 11.0 \\
\hline 103 & SSV2008125 & 70.0 & 0.0 & 7.0 & 78.2 & 0.0 & 9.0 & 123.7 & 0 & 10 & 145.9 & 0.0 & 11.0 \\
\hline 104 & SSV2008181 & 56.5 & 0.0 & 8.0 & 69.1 & 0.0 & 9.5 & 122.2 & 0 & 9 & 156.9 & 0.0 & 10.0 \\
\hline Mean & & 67.15 & 0.33 & 7.591 & 77.23 & 0.322 & 9.51 & 110.37 & 0.32 & 9.76 & 130.6 & 0.558 & 11.5 \\
\hline SE & & 1.633 & 0.07 & 0.0206 & 0.05946 & 0.0068 & 0.0136 & 0.25 & 0.091 & 0.0123 & 0.0951 & 0.34 & 0.490 \\
\hline LSD ( & & 4.58 & 0.05 & 0.4588 & 0.16677 & 0.8847 & 0.8514 & 9.23 & 0.83 & 15.23 & 5.90 & 1.3251 & 1.210 \\
\hline
\end{tabular}

Plht=plant height; TIno.=number of tillers; Lfno.- number of leaves; WAI= week after inoculation 
Table 2. Mean of number of days to $50 \%$ flowering, days to $50 \%$ heading and grain yield of sorghum genotypes screened for head smut

\begin{tabular}{|c|c|c|c|c|c|}
\hline $\mathbf{S} / \mathbf{N}$ & $\begin{array}{l}\text { Sorghum } \\
\text { genotype }\end{array}$ & $\begin{array}{l}\text { Days to } \\
\text { flowering }\end{array}$ & $50 \%$ & $\begin{array}{l}\text { Days to } 50 \% \\
\text { heading }\end{array}$ & $\begin{array}{c}\text { Grain yield } \\
(\mathrm{kg} / \mathrm{ha})\end{array}$ \\
\hline 1 & SSV2006002* & 67 & & 98 & 231.8 \\
\hline 2 & SSV2006006 & 70 & & 98 & 340.0 \\
\hline 3 & SSV2006007 & 72 & & 92 & 280.0 \\
\hline 4 & SSV2006011 & 76 & & 102 & 351.0 \\
\hline 5 & SSV2006013* & 80 & & 100 & 145.1 \\
\hline 6 & SSV2006014 & 76 & & 97 & 238.9 \\
\hline 7 & SSV2006015 & 79 & & 104 & 450.9 \\
\hline 8 & SSV2006016 & 90 & & 120 & 560.0 \\
\hline 9 & SSV2006017 & 97 & & 112 & 439.0 \\
\hline 10 & SSV2006018 & 87 & & 113 & 460.0 \\
\hline 11 & SSV2006021* & 100 & & 140 & 301.1 \\
\hline 12 & SSV2006024 & 89 & & 111 & 512.2 \\
\hline 13 & SSV2006026* & 76 & & 100 & 341.1 \\
\hline 14 & SSV2006027 & 78 & & 120 & 321.9 \\
\hline 15 & SSV2006029 & 67 & & 110 & 421.9 \\
\hline 16 & SSV2006030* & 82 & & 109 & 331.1 \\
\hline 17 & SSV2006031* & 79 & & 98 & 231.9 \\
\hline 18 & SSV2006033 & 81 & & 95 & 372.0 \\
\hline 19 & SSV2006035 & 71 & & 98 & 412.9 \\
\hline 20 & SSV2006036 & 68 & & $10^{`} 2$ & 325.8 \\
\hline 21 & SSV2006039 & 66 & & 108 & 421.9 \\
\hline 22 & SSV2006041 & 64 & & 105 & 430.0 \\
\hline 23 & SSV2006045 & 81 & & 114 & 351.9 \\
\hline 24 & SSV2006047 & 69 & & 108 & 351.9 \\
\hline 25 & SSV2008001 & 80 & & 92 & 365.9 \\
\hline 26 & SSV2008002* & 84 & & 99 & 123.2 \\
\hline 27 & SSV2008004* & 76 & & 98 & 143.1 \\
\hline 28 & SSV2008005 & 83 & & 97 & 451.9 \\
\hline 29 & SSV2008006* & 100 & & 95 & 102.1 \\
\hline 30 & SSV2008007 & 91 & & 100 & 345.2 \\
\hline 31 & SSV2008008 & 86 & & 104 & 451.9 \\
\hline 32 & SSV2008009 & 101 & & 100 & 357.0 \\
\hline 33 & SSV2008010* & 91 & & 97 & 261.9 \\
\hline 34 & SSV2008012 & 67 & & 104 & 348.9 \\
\hline 35 & SSV2008013 & 89 & & 120 & 560.2 \\
\hline 36 & SSV2008017 & 87 & & 112 & 579.0 \\
\hline 37 & SSV2008018 & 89 & & 113 & 451.0 \\
\hline 38 & SSV2008019 & 67 & & 140 & 287.9 \\
\hline 39 & SSV2008021 & 65 & & 111 & 600.9 \\
\hline 40 & SSV2008022 & 70 & & 100 & 567.2 \\
\hline 41 & SSV2008023 & 80 & & 120 & 431.0 \\
\hline 42 & SSV2008025 & 68 & & 110 & 154.7 \\
\hline 43 & SSV2008026* & 59 & & 109 & 212.2 \\
\hline 44 & SSV2008028 & 65 & & 98 & 234.2 \\
\hline 45 & SSV2008029* & 63 & & 95 & 217.2 \\
\hline 46 & SSV2008030* & 69 & & 98 & 356.1 \\
\hline 47 & SSV2008031* & 72 & & 102 & 569.2 \\
\hline 48 & SSV2008032 & 74 & & 108 & 450.0 \\
\hline 49 & SSV2008033* & 76 & & 87 & 430.0 \\
\hline 50 & SSV2008034* & 78 & & 99 & 154.1 \\
\hline 51 & SSV2008035* & 78 & & 98 & 345.0 \\
\hline 52 & SSV2008036 & 80 & & 97 & 345.0 \\
\hline 53 & SSV2008039 & 80 & & 95 & 432.0 \\
\hline 54 & SSV2008040 & 80 & & 98 & 543.0 \\
\hline 55 & SSV2008041* & 90 & & 99 & 113.1 \\
\hline 56 & SSV2008042 & 81 & & 103 & 367.0 \\
\hline 57 & SSV2008044 & 80 & & 100 & 621.0 \\
\hline 58 & SSV2008046 & 97 & & 119 & 450.0 \\
\hline 59 & SSV2008047 & 78 & & 109 & 234.1 \\
\hline 60 & SSV2008048* & 69 & & 104 & 130.0 \\
\hline
\end{tabular}




\begin{tabular}{|c|c|c|c|c|c|}
\hline $\mathbf{S} / \mathbf{N}$ & $\begin{array}{l}\text { Sorghum } \\
\text { genotype }\end{array}$ & $\begin{array}{l}\text { Days to } \\
\text { flowering }\end{array}$ & $50 \%$ & $\begin{array}{l}\text { Days to } 50 \% \\
\text { heading }\end{array}$ & $\begin{array}{c}\text { Grain yield } \\
(\mathrm{kg} / \mathrm{ha})\end{array}$ \\
\hline 61 & SSV2008049 & 80 & & 120 & 378.0 \\
\hline 62 & SSV2008051* & 89 & & 112 & 214.1 \\
\hline 63 & SSV2008052 & 78 & & 113 & 412.9 \\
\hline 64 & SSV2008053 & 69 & & 140 & 540.0 \\
\hline 65 & SSV2008054 & 70 & & 111 & 542.0 \\
\hline 66 & SSV2008055 & 78 & & 100 & 456.9 \\
\hline 67 & SSV2008056 & 79 & & 120 & 453.0 \\
\hline 68 & SSV2008057 & 78 & & 110 & 432.0 \\
\hline 69 & SSV2008058 & 69 & & 109 & 345.9 \\
\hline 70 & SSV2008059 & 78 & & 98 & 600.0 \\
\hline 71 & SSV2008061 & 70 & & 95 & 541.0 \\
\hline 72 & SSV2008063 & 78 & & 98 & 430.0 \\
\hline 73 & SSV2008064 & 69 & & 102 & 430.9 \\
\hline 74 & SSV2008066* & 69 & & 108 & 421.0 \\
\hline 75 & SSV2008067 & 70 & & 877 & 453.9 \\
\hline 76 & SSV2008070 & 79 & & 99 & 430.0 \\
\hline 77 & SSV2008072* & 73 & & 98 & 321.9 \\
\hline 78 & SSV2008074 & 78 & & 97 & 340.0 \\
\hline 79 & SSV2008075 & 84 & & 95 & 541.1 \\
\hline 80 & SSV2008076 & 83 & & 98 & 450.9 \\
\hline 81 & SSV2008078 & 78 & & 99 & 321.0 \\
\hline 82 & SSV2008079* & 76 & & 103 & 438.0 \\
\hline 83 & SSV2008080* & 78 & & 100 & 145.5 \\
\hline 84 & SSV2008082 & 69 & & 100 & 165.1 \\
\hline 85 & SSV2008084 & 90 & & 110 & 520.0 \\
\hline 86 & SSV2008085 & 78 & & 102 & 456.8 \\
\hline 87 & SSV2008086 & 80 & & 107 & 437.0 \\
\hline 88 & SSV2008087* & 87 & & 97 & 560.0 \\
\hline 89 & SSV2008088* & 89 & & 99 & 567.1 \\
\hline 90 & SSV2008089 & 87 & & 100 & 101.1 \\
\hline 91 & SSV2008090 & 79 & & 120 & 451.0 \\
\hline 92 & SSV2008094 & 80 & & 114 & 348.0 \\
\hline 93 & SSV2008096 & 80 & & 112 & 453.0 \\
\hline 94 & SSV2008100 & 78 & & 111 & 345.0 \\
\hline 95 & SSV2008101 & 80 & & 114 & 437.9 \\
\hline 96 & SSV2008107 & 68 & & 112 & 450.0 \\
\hline 97 & SSV2008110 & 68 & & 111 & 341.9 \\
\hline 98 & SSV2008111 & 78 & & 110 & 398.0 \\
\hline 99 & SSV2008112* & 87 & & 102 & 119.0 \\
\hline 100 & SSV2008113 & 67 & & 107 & 278.0 \\
\hline 101 & SSV2008116 & 98 & & 97 & 450.0 \\
\hline 102 & SSV2008117 & 76 & & 99 & 560.0 \\
\hline 103 & SSV2008125 & 76 & & 100 & 367.9 \\
\hline \multirow[t]{4}{*}{104} & SSV2008181* & 80 & & 120 & 376.3 \\
\hline & Mean & 78.5 & & 105.27 & 376.3 \\
\hline & S.E. & 0 & & 0.4903 & 377.03 \\
\hline & LSD (0.05) & 3.21 & & 1.3751 & 2.49 \\
\hline
\end{tabular}

* indicates infected genotype

\section{REFERENCES}

Ahliawate, M. S. (2007) Pathogenicity and aetiology of Fusarium species associated with Pokkahboeng disease on Sugarcane. Unpublished M. Sc. Thesis Universiti Sains Malaysia. Pp 23-26

David, G. and Adam, P (1998) Crops of the drier regions of the tropics. Education low price edition. Pp90-98

FAO (2010) Food and Agricultural Organization Statistics. Available @www.fao.org//faostat
Frederiksen R.A. (1977) Head smuts of corn and sorghum. Proceedings, $32^{\text {nd }}$ Annual corn and Sorghum Research Conference 32:89-105

ICRISAT (1978) Information bulletin No. 2. Sorghum and Pearl millet identification hand book edited by Williams, R.J., R.A.,Frederiksen and J.C.,Girard.

IPM (2008) Integrated pest management . Reports on plant disease (RPD) No.408. Sorghum smuts Pp 1-6 
Komolafe, M.F., Adegbola,A.A.,Are,A.L. and Ashaye, T.J. (1985) Agricultural Science for West African Schools and Colleges. $2^{\text {nd }}$ edition.University Press Limited, Ibadan, pp20-120

Keay, M.A.,Dransfield,M., McDonald,D., kING, S.B.,Fowler,A.M.,Quanash, S.K. and Carter, J.B.H. (1969) Plant pathology section. Report of the Institute of Agricultural Reseachr, Samaru. 1967-68. 52-61

King, S.B. (1969) Reports on the Advisory Board on the Institute (IAR-Samaru) work in 1969. Crops and animals pp14-15

Kutama, A.S., Aliyu, B.S., and Emechebe, A.M. (2011a): Field screening of sorghum genotypes for resistance to head smut in the Sudan savanna agro-ecological zone of Nigeria. In: Proceedings of the International Conference on science and Technology held @ Hotel Dona, Porto Novo, Republic of Benin 1(7): $90-98$

Kutama, A.S., Emechebe, A.M; and Aliyu, B.S (2011b) Field evaluation of some inoculation techniques on the incidence and severity of sorghum head smut (Sporisoriumreilianum) in Nigerian Sudan savanna. Biological and Environmental Sciences Journal for the Tropics, 8(3):292- 296

Kutama, A.S., Aliyu, B.S., and Emechebe, A.M. (2011c) Screening of sorghum genotypes for resistance to loose smut in Nigeria.Bayero Journal of Pure and Applied Sciences 4(2):199-203

Kutama, A.S (2012): Studies on the epidemiology and control of head and loose smuts of sorghum in the sudan savanna region of Nigeria. Ph.D Thesis (unpublished), Department of Plant Science, Bayerouniversity, Kano, Nigeria. P165-180

Louis, K.P.,Ndiaga, C. Ousmane,Ndoye (2007). Assessing the vulnerability of selected Sorghum line from the united states of America to long smut (SoroposporiumehrenberghiNanky). Crop Protection 26: 1771- 1776

Matheussen,. A.M..; P.W.Morgan and R.A.Frederiksen (1991) implication of Gibbrellins in head smut ( sporisorium relianum0 of sorghum Sorghumbicolor. Plantphysiol. 96:537-544

Mehrotra, R.S., and Aggarwal, A. (2003) Plant pathology, Second edition. Tata McGrawHill Publishing Company Limited. pp 411-423
Morgan, C. Miller, F.R., Quinby, J.R.(1977) Manipulation Of Sorghum Growth With Gibbrellic Acid. Agron. .J. 69:789-793

Naidoo,C.,Morgan, P.W. Collins, A.C., Fredriksen, R.A. (1991) Effect of Sporisorium reilianum on plant height and internode elongation in sorghumbicolor in naturally infected field. Sorghum news letter 31, London. PP 101- 110

Norman, M.J.T.,Pearson,C.J. and Searle, P.G.E. (1984) Sorghum In: the Ecology of Tropical Food Crops. Pp120-136. Cambridge University Press

Phinney, B.O., Spray, C. (1982) Chemical genetics and the giggrellin pathway in Zea mays L. In P.F.Wareing, ed, Plant growth Substances. Academic Press,

Tarr, S.A.J. (1962) Diseases of Sorghum, Sudan grass and Browncorn.Commonwealth Mycological Institute, Kew, Survey Pp171-247

Singh, R. S. (2005) introduction to the principle of Plant pathology $4^{\text {th }}$ edition. Oxford and IBH Publishing Co. PVT. LTD New Delhi .Pp.11512 